\title{
RECENT CLASSIFICATION \\ AND CHARACTERIZATION RESULTS IN GEOMETRIC TOPOLOGY
}

\author{
J. J. DIJKSTRA, T. DOBROWOLSKI, W. MARCISZEWSKI, \\ J. VAN MILL, AND J. MOGILSKI
}

\begin{abstract}
We announce a complete topological classification of the function spaces $C_{p}(X)$ of Borel class not higher than 2 , provided that $X$ is a countable space. We also present a topological classification of the $k$-dimensional universal pseudoboundaries and pseudointeriors in $\mathbf{R}^{n}$, and we investigate under what conditions strong negligibility of $\sigma Z$-sets characterizes Hilbert space manifolds.
\end{abstract}

\section{INTRODUCTION}

The work presented in this announcement traces its history back to Fréchet [18] and Banach [6] who proposed to classify metric linear spaces according to topological type. For complete spaces this program was carried out by Anderson [1], Kadec [21], and Toruńczyk [25]: A Fréchet space is characterized topologically by its linear dimension (i.e., minimal cardinality of sets with a dense span). The classification of incomplete linear spaces, however, is still in the beginning stage. In the case that the space is a so-called absorber (see $\S 2$ ) characterizations have been developed (Mogilski [24] and Bestvina and Mogilski [8]). We apply these results to the classification of certain function spaces. If $X$ is a space, then $C_{p}(X)$ denotes the space of continuous, real valued functions on $X$ endowed with the topology of pointwise convergence. This function space is metrizable only if $X$ is a countable space (barring spaces without point separating real valued functions). Therefore we consider countable completely regular spaces $X$ that are for obvious reasons also nondiscrete. We show that all $F_{\sigma \delta}$-spaces

Received by the editors September 28, 1989 and, in revised form November 30, 1989.

1980 Mathematics Subject Classification (1985 Revision). Primary 57N15, $57 \mathrm{~N} 20$. 
$C_{p}(X)$ are homeomorphic to $\sigma^{\omega}$, the countable product of $l_{f}^{2}=$ $\left\{\left(x_{i}\right) \in l^{2}: x_{i}=0\right.$ for almost all $\left.i\right\}$. According to Dijkstra et al. [14], $C_{p}(X)$ cannot be an absolute $G_{\delta \sigma}$-set and hence we obtain a complete classification of the spaces $C_{p}(X)$ of Borel class not higher than 2. Using similar techniques, we detect other sequence and function spaces homeomorphic to $\sigma^{\omega}$.

It was observed by Geoghegan and Summerhill [20] that the techniques developed for infinite-dimensional manifolds can be applied to $\mathbf{R}^{n}$ as well. They constructed $k$-dimensional universal pseudoboundaries $B_{k}^{n}$ and pseudointeriors $s_{k}^{n}$ in $\mathbf{R}^{n}$ analogously to the pseudoboundary $B$ and the pseudointerior $s$ of the Hilbert cube. Pseudoboundaries are absorbers and pseudointeriors are their complements. The pseudointeriors $s_{k}^{n}$ for $n \geq 2 k+1$ are particularly interesting because of their resemblance to the separable Hilbert space $l^{2}$, which is homeomorphic to the pseudointerior $s$ by a celebrated theorem of Anderson [1]. We show that $B_{k}^{n}$ is homeomorphic to $B_{k}^{m}$ if and only if $s_{k}^{n}$ is homeomorphic to $s_{k}^{m}$ if and only if $n=m$ or $n, m \geq 2 k+1$.

In the final section we consider Hilbert space manifolds and strongly negligible sets. A strongly negligible subset of a space is a set whose complement is topologically equivalent to the whole space via homeomorphisms that can be chosen arbitrarily close to the identity. This concept is closely related to absorbers (see Chapter 1 in [9]). Anderson shows in [2] that in Hilbert space manifolds the strongly negligible sets coincide with the $\sigma$ Z-sets. We discuss the result that this property characterizes Hilbert space manifolds if every compactum is a strong Z-set but not if every compactum is merely a Z-set.

For background information on infinite-dimensional topology see [7] or [23].

\section{ABSORBERS}

The central idea in this note is the concept of a (generalized) absorber. Let $X$ be a space and let $\mathscr{M}$ be a collection of closed subsets that is hereditary and additive. A countable union $A$ of elements of $\mathscr{M}$ is called an $\mathscr{M}$-absorber if for every $D \in \mathscr{M}$ and every collection $\mathscr{U}$ of open subsets of $X$ there is a homeomorphism $h$ of $X$ that is $\mathscr{U}$-close to the identity and that has the property $h(D \cap \cup \mathscr{U}) \subseteq A$. This definition is due to West [27].

This idea was extended as follows by Bestvina and Mogilski [8]. 
Let $\mathscr{K}$ be a class of spaces that is topological (i.e., homeomorphic images of elements of $\mathscr{K}$ belong to $\mathscr{K}$ ), additive, and hereditary with respect to closed sets. A subset $X$ of a topological copy $E$ of $l^{2}$ is a generalized $\mathscr{K}$-absorber if:

(1) $X=\bigcup_{i=1}^{\infty} X_{i}$, where each $X_{i} \in \mathscr{K}$ is a Z-set in $X$, and

(2) given an open cover $\mathscr{U}$ of $X$ in $E$, a set $D \in \mathscr{K}$, a closed subset $C$ of $D$, and a map $f: D \rightarrow \bigcup \mathscr{U}$ such that $f \mid C$ is a $Z$-embedding into $X$, there exists a Z-embedding $v: D \rightarrow X$ that is $\mathscr{U}$-close to $f$ and that satisfies $v \mid C=$ $f \mid C$.

A closed subset $S$ of a space $X$ is called a $Z$-set if continuous maps from $X$ into $X \backslash S$ can be found arbitrarily close to the identity. A Z-embedding is an embedding whose range is a Z-set and a $\sigma Z$-set is a countable union of Z-sets.

Bestvina and Mogilski [8] proved that any two generalized $\mathscr{K}$ absorbers in $E$ are homeomorphic. We are especially interested in the case where $\mathscr{K}$ is the class $\mathscr{F}_{\sigma \delta}$ of all absolute $F_{\sigma \delta}$-spaces. Recall that the first Borel class consists of the absolute $F_{\sigma}$-spaces ( $=$ $\sigma$-compacta) and the absolute $G_{\delta}$-spaces ( = completely metrizable spaces). The second Borel class then consists of $\mathscr{F}_{\sigma \delta}=\{$ countable intersections of elements of $\left.\mathscr{F}_{\sigma}\right\}$ and $\mathscr{G}_{\delta \sigma}=\{$ countable unions of elements of $\left.\mathscr{G}_{\delta}\right\}$. In our case the space $\sigma^{\omega}$ is the standard generalized $\mathscr{F}_{\sigma \delta}$-absorber and we have:

Proposition 2.1. A space $X$ is homeomorphic to $\sigma^{\omega}$ if and only if (a) $X$ is an absolute retract; (b) $X=\bigcup_{i=1}^{\infty} X_{i}$, where each $X_{i} \in \mathscr{F}_{\sigma \delta}$ is a Z-set in $X$; (c) $X$ is homeomorphic to a space $Y$ such that $X_{f}^{\omega} \subseteq Y \subseteq X^{\omega}$; and (d) $X$ contains a closed subset homeomorphic to $X^{\omega}$.

In this proposition $X_{f}^{\omega}$ stands for the subset of the product $X^{\omega}$ consisting of the sequences $\left(x_{i}\right)$ that have almost all terms equal to some fixed point in $X$. Proposition 2.1 can be used to prove the following:

Theorem 2.2. The following linear spaces are homeomorphic:

(1) $\sigma^{\omega}=\left(l_{f}^{2}\right)^{\omega}$;

(2) $c_{0}=\left\{\left(x_{i}\right) \in \mathbf{R}^{\omega}: x_{i} \rightarrow 0\right\}$ endowed with the topology of coordinatewise convergence;

(3) $\tilde{l}^{p}=\bigcap_{q>p} l^{q},(0 \leq p<\infty)$, with the topology of coordinatewise convergence; 
(4) $\tilde{L}^{p}=\bigcap_{q<p} L^{q},(0<p \leq \infty)$, with the topology of convergence in (Lebesgue) measure.

Details will appear in [15].

\section{FunCtion SPACES IN THE TOPOLOGY OF POINTWISE CONVERGENCE}

Using a slight modification of Proposition 2.1 and some elements of the theory of Borel filters, we prove:

Theorem 3.1. Let $X$ be a nondiscrete, countable, completely regular space such that the function space $C_{p}(X)$ is an absolute $F_{\sigma \delta}$-set. Then $C_{p}(X)$ and $C_{p}^{*}(X)$ are homeomorpic to $\sigma^{\omega}$.

Here $C_{p}^{*}(X)$ is the subspace of $C_{p}(X)$ consisting of all bounded functions. Details will appear in [17]. Since for every countable metric space $X$ the function space $C_{p}(X)$ is in $\mathscr{F}_{\sigma \delta}$, this theorem generalizes results of van Mill [22], Baars et al. [5], and Dobrowolski et al. [16]. As an application of Theorem 3.1 we can answer in the negative some questions of Arhangel'skii $[3,4]$ by producing a countable, completely regular space $X$ which fails to be a $b_{\mathbf{R}}$-space, a $k$-space, and an $\aleph_{0}$-space, while the function space $C_{p}(X)$ is homeomorphic to the $C_{p}$ of the convergent sequence.

\section{Classification OF FINITE-Dimensional PSEUDOBOUNDARIES AND PSEUDOINTERIORS}

Let $n$ and $k$ be fixed integers such that $n \geq 1$ and $0 \leq k<$ $n$. In addition, let $\mathscr{M}_{k}^{n}$ denote the collection of "tame" $\leq k$ dimensional compacta in $\mathbf{R}^{n}$. In [20] Geoghegan and Summerhill prove that there exists an $\mathscr{M}_{k}^{n}$-absorber. This set is called the $k$-dimensional universal pseudoboundary of $\mathbf{R}^{n}$, and we denote it by $B_{k}^{n}$. The $k$-dimensional universal pseudointerior $s_{k}^{n}$ is the complement of $B_{n-k-1}^{n}$ in $\mathbf{R}^{n}$. If $n \geq 2 k+1$, then $s_{k}^{n}$ can be seen as a $k$-dimensional analogue of Hilbert space in the topological category. These spaces are $k$-dimensional (locally) $(k-1)$-connected complete spaces which are universal for the $k$ dimensional spaces and which share the following properties with Hilbert space: (a) homogeneity, (b) every $\sigma$ Z-set is strongly negligible, and (c) Toruńczyk's discrete approximation property. We classify these spaces topologically by deriving the following:

Theorem 4.1. $B_{k}^{n}$ is homeomorphic to $B_{k}^{m}$ if and only if $s_{k}^{n}$ is homeomorphic to $s_{k}^{m}$ if and only if $n=m$ or $n, m \geq 2 k+1$. 
The following consequence is noteworthy. Consider the trefoil (or any other knot) in $\mathbf{R}^{3}$. Since the trefoil and the unknot are elements of $\mathscr{M}_{1}^{3}$, we may assume that they are subsets of $s_{1}^{3}$ or $B_{1}^{3}$. Since tame embeddings of $S^{1}$ are equivalent in $\mathbf{R}^{4}$, they are equivalent in $s_{1}^{4}$ and $B_{1}^{4}$ (see [19, Theorem 2.5] and [9, Theorem 1.2.13]). The theorem then implies that the trefoil is unknotted in both $s_{1}^{3}$ and $B_{1}^{3}$.

The method used in [13] to prove the theorem is strongly "infinite-dimensional" in spirit and is based on techniques similar to those that were used in $\S 2$, in [8], and in [24]. In order to show that $B_{k}^{m}$ is homeomorphic to $B_{k}^{n}$ for $m, n \geq 2 k+1$, we use as a fixed model for these spaces a $k$-dimensional absorber $B_{k}^{\omega}$ in the Hilbert space $l^{2}$. The existence of $B_{k}^{\omega}$ is established in [10]. Let $C$ stand for the cone of $l^{2}$ and let $\pi$ be the projection $\mathbf{R}^{n} \times C \rightarrow$ $\mathbf{R}^{n}$. We embed $B_{k}^{\omega}$ as a $k$-absorber in the topological Hilbert space $\mathbf{R}^{n} \times C$ in such a way that $\pi\left(B_{k}^{\omega}\right)=B_{k}^{n}$. Using a version of Bing's shrinking criterion that was developed by Torunczyk [26] for incomplete spaces, we prove that $\pi \mid B_{k}^{\omega}: B_{k}^{\omega} \rightarrow B_{k}^{n}$ is a near homeomorphism if $n \geq 2 k+1$.

The corresponding statement for $s_{k}^{n}$ follows easily. The pseudointeriors $s_{k}^{m}$ and $s_{k}^{n}$ contain embedded copies of $B_{k}^{m}$ and $B_{k}^{n}$, respectively. By a classic Theorem of Lavrentiev the homeomorphism between $B_{k}^{m}$ and $B_{k}^{n}$ can be extended to a homeomorphism between $G_{\delta}$-subsets $X$ and $Y$ of $s_{k}^{m}$ and $s_{k}^{n}$, respectively. Since it can be shown that the complements of $X$ and $Y$ are strongly negligible, we have a homeomorphism between $s_{k}^{m}$ and $s_{k}^{n}$.

\section{Characterizing Hilbert space topology} IN TERMS OF STRONG NEGLIGIBILITY

In the late 1960s R. D. Anderson introduced the concept of a strongly negligible set to infinite-dimensional topology. He shows in [2] that in Hilbert space manifolds the strongly negligible sets are precisely the $\sigma \mathrm{Z}$-sets. Let us denote this property by $\mathrm{SN}=\sigma \mathrm{Z}$. We investigate under what conditions the property characterizes the Hilbert space manifolds among the complete absolute neighbourhood retracts (ANRs). In [11] we proved the following:

Theorem 5.1. A complete ANR is a Hilbert space manifold if and only if $S N=\sigma \mathrm{Z}$ and moreover if every compact subset is a strong Z-set. 
A closed subset $S$ of a space $X$ is called a strong Z-set if there exist continuous maps $f: X \rightarrow X$, arbitrarily close to the identity, such that the closure of $f(X)$ does not meet $S$ (cf. the definition of Z-set in $\S 2$ ). This theorem is sharp in the sense that there exists an absolute retract, not homeomorphic to $l^{2}$, with $\mathrm{SN}=\sigma \mathrm{Z}$ and the property that compacta are Z-sets rather than strong Z-sets. This counterexample will be described in [12].

\section{REFERENCES}

1. R. D. Anderson, Hilbert space is homeomorphic to the countable infinite product of lines, Bull. Amer. Math. Soc. 72 (1966), 515-519.

2. Strongly negligible sets in Fréchet manifolds, Bull. Amer. Math. Soc. 75 (1969), 64-67.

3. A. V. Arhangel'skii, A survey of $C_{p}$-theory, Questions Answers Gen. Topology 5 (1987), 1-109.

4. __ Some results and problems in $C_{p}(X)$-theory, Proc. Sixth Prague Topological Sympos., Heldermann, Berlin, 1988, pp. 11-31.

5. J. Baars, J. de Groot, J. van Mill, and J. Pelant, On topological and linear homeomorphisms of certain function spaces, Topology Appl. 32 (1989), 267-277.

6. S. Banach, Théorie des Opérations Linéaires, PWN, Warsaw, 1932.

7. C. Bessaga and A. Pelczyński, Selected topics in infinite-dimensional topology, PWN, Warsaw, 1975.

8. M. Bestvina and J. Mogilski, Characterizing certain incomplete infinite-dimensional absolute retracts, Michigan Math. J. 33 (1986), 291-313.

9. J. J. Dijkstra, Fake topological Hilbert spaces and characterizations of dimension in terms of negligibility, CWI Tracts vol. 2, Centre for Math. and Comp. Sci., Amsterdam, 1984.

10. _ , k-Dimensional skeletoids in $\mathbf{R}^{n}$ and the Hilbert cube, Topology Appl. 19 (1985), 13-28.

11. Strong negligibility of $\sigma$-compacta does not characterize Hilbert space, Pacific J. Math. 127 (1987), 19-30.

12. _ Characterizing Hilbert space topology in terms of strong negligibility, preprint.

13. J. J. Dijkstra, J. van Mill, and J. Mogilski, Classification of finite-dimensional universal pseudoboundaries and pseudointeriors, Trans. Amer. Math. Soc. (to appear).

14. J. J. Dijkstra, T. Grilliot, D. Lutzer, and J. van Mill, Function spaces of low Borel complexity, Proc. Amer. Math. Soc. 94 (1985), 703-710.

15. T. Dobrowolski and J. Mogilski, Certain sequence and function spaces homeomorphic to the countable product of $l_{f}^{2}$, preprint.

16. T. Dobrowolski, S. P. Gulko, and J. Mogilski, Function spaces homeomorphic to the countable product of $l_{2}^{f}$, Topology Appl. (to appear).

17. T. Dobrowolski, W. Marciszewski, and J. Mogilski, On topological classifcation of function spaces $C_{p}(X)$ of low Borel complexity, preprint. 
18. M. Fréchet, Les espaces abstraits, Hermann, Paris, 1928.

19. R. Geoghegan and R. R. Summerhill, Concerning the shapes of finite-dimensional compacta, Trans. Amer. Math. Soc. 179 (1973), 281-292.

20. __ Pseudoboundaries and pseudointeriors in euclidean spaces and topological manifolds, Trans. Amer. Math. Soc. 194 (1974), 141-165.

21. M. I. Kadec, A proof of topological equivalence of all separable infinite-dimensional Banach spaces (Russian), Funkcional. Anal. i Prilozen. 1 (1967), 53-62.

22. J. van Mill, Topological equivalence of certain function spaces, Compositio Math. 63 (1987), 159-188.

23. __ Infinite-dimensional Topology: Prerequisites and introduction, NorthHolland, Amsterdam, 1989.

24. J. Mogilski, Characterizing the topology of infinite-dimensional $\sigma$-compact manifolds, Proc. Amer. Math. Soc. 92 (1984), 111-118.

25. H. Toruńczyk, Characterizing Hilbert space topology, Fund. Math. 111 (1981), 247-262.

26. A A correction of two papers concerning Hilbert manifolds, Fund. Math. 125 (1985), 89-93.

27. J. E. West, The ambient homeomorphy of an incomplete subspace of infinite-dimensional Hilbert spaces, Pacific J. Math. 34 (1970), 257-267.

Department of Mathematics, The University of Alabama, Box 870350, Tuscaloosa, Alabama 35487-0350, USA (J. J. Dijkstra and J. Mogilski)

E-mail:JDIJKSTR@UA1VM.UA.EDU

E-mail:JMOGILSK@UA1VM.UA.EDU

Instytut Matematyki, Uniwersytet Warszawski, PKiN, 00-901 Warszawa, Poland (T. Dobrowolski)

Department of Mathematics, The University of Kansas, Lawrence, KANSAS 66045, USA (W. MARCISZEWSKI)

Faculteit Wiskunde en Informatica, Vrije Universiteit, Postbus 7161 , 1007 MC AMSTerdam, The Netherlands (J. Van MiLl)

E-mail:VANMILL@CS.VU.NL 
